This item was submitted to Loughborough's Research Repository by the author.

Items in Figshare are protected by copyright, with all rights reserved, unless otherwise indicated.

\title{
Comparison of DC and RF sputtered aluminium-doped zinc oxide for photovoltaic applications
}

PLEASE CITE THE PUBLISHED VERSION

http://dx.doi.org/10.1109/PVSC.2015.7355900

PUBLISHER

(C) IEEE

VERSION

AM (Accepted Manuscript)

\section{PUBLISHER STATEMENT}

This work is made available according to the conditions of the Creative Commons Attribution-NonCommercialNoDerivatives 4.0 International (CC BY-NC-ND 4.0) licence. Full details of this licence are available at: https://creativecommons.org/licenses/by-nc-nd/4.0/

\section{LICENCE}

CC BY-NC-ND 4.0

\section{REPOSITORY RECORD}

Isherwood, Patrick J.M., Michael Gona, Jake W. Bowers, Nuno N.P. Neves, Paul Newbatt, and Michael Walls. 2019. "Comparison of DC and RF Sputtered Aluminium-doped Zinc Oxide for Photovoltaic Applications". figshare. https://hdl.handle.net/2134/20174. 


\title{
Comparison of DC and RF sputtered aluminium-doped zinc oxide for photovoltaic applications
}

\author{
Patrick J. M. Isherwood*, Michael Gona*, Jake W. Bowers*, Nuno Neves ${ }^{\dagger}$, Paul Newbatt \\ and John M. Walls* \\ * CREST, Loughborough University, Loughborough, Leicestershire, LE11 3TU, UK \\ † Innovnano SA, IParque Lote 13, Coimbra, Antanhol 3040-570, Portugal
}

\begin{abstract}
AZO was sputter deposited using both RF and DC power supplies from a pre-formed ceramic AZO target with a 2 wt \% aluminium oxide dopant concentration. The target was formed by hot pressing pre-doped nanoparticles produced using an emulsion detonation synthesis technique. AZO films were found to display good optical and electrical properties regardless of deposition technique. Increased temperatures above $200{ }^{\circ} \mathrm{C}$ were found to have a negative impact on the electrical properties, with a corresponding increase in infrared transmission. Visible transmission was found to be good (above $79 \%$ ) for all films except those deposited using DC power at room temperature. The DC sputtering process was stable and was found to approximately double the deposition rate with no corresponding drop in film quality for films deposited at moderate temperatures. This makes DC deposition more industrially attractive when process temperature is not a concern. For room temperature depositions, RF was found to produce superior transmission and electrical characteristics.
\end{abstract}

Index Terms-Wide band gap semiconductors, II-VI semiconductor materials, thin films, conductive films.

\section{INTRODUCTION}

Transparent conducting oxides (TCOs) are degenerately doped wide band gap metal oxide semiconductors, and are widely used for a range of applications including computer screen electrodes, touch-screen panels, mobile phone screens, LED contacts, and as top contact layers in thin film photovoltaic cells [1], [2], [3]. They are easy to synthesise and deposit, with a wide range of different deposition techniques being regularly employed [3]. Significant concerns regarding their use include a tendency to crack when flexed, their typical trade-off between optical transparency and conductivity, and in some cases cost issues resulting from the use of relatively rare and expensive raw materials [3], [4]. At present, the most widely used TCOs are tin-doped indium oxide (ITO) and fluorine-doped tin oxide (FTO). ITO is increasingly expensive because of indium scarcity issues coupled with high demand [5]. FTO has some applicability issues as there are a limited number of ways in which it can be deposited due to the difficulty of working with fluorine [3]. It is typically deposited onto high-temperature glass as it exits the float line by chemical vapour deposition [3].

Aluminium-doped zinc oxide (AZO) is a promising alternative material, and has been shown by numerous groups to have excellent optical and electrical properties [3], [5]. It consists entirely of earth-abundant elements, and can be deposited using a wide range of techniques including sputtering, chemical vapour deposition, spray coating and chemical bath deposition [3], [6]. Despite being a highly promising alternative, AZO has some limitations which may prevent its use in specific applications. Most notably, it is known to have some chemical stability issues, and can degrade in highly humid environments if not properly encapsulated. This can also be an advantage, as it means that it is easier to etch and can easily be textured [3], [7]. It has significant potential as an alternative to both ITO and FTO as a transparent front contact material for photovoltaic devices.

Innovnano have developed a new emulsion detonation synthesis technique for the production of pre-doped AZO nanoparticles which can then be hot-pressed to form sputter targets. The two most significant advantages of this technique are the ability to produce material with the precise doping concentration required, and that because it synthesises predoped nanoparticles, the dopant is spread homogeneously throughout the target. The present work compares the effects of DC and RF sputtering on the deposition rate using a 2 w.t $\%$ aluminium oxide doped nanoparticulate target and examines the structural, electrical and optical properties of the resulting thin films.

\section{EXPERIMENTAL DETAILS}

AZO films were deposited onto pre-cleaned $5 \mathrm{~cm} \mathrm{x} 5 \mathrm{~cm}$ soda lime glass slides by sputtering from a pre-formed ceramic 2 wt. \% aluminium oxide doped AZO target. Deposition was carried out using an AJA International Orion $8 \mathrm{HV}$ sputter coater equipped with an AJA 600 series RF power supply and an Advanced Energy MDX 500 DC power supply. Argon flow rate was maintained at $5 \mathrm{SCCM}$, and the process pressure was 1 mTorr $(0.133 \mathrm{~Pa})$. The applied power used was 180 $\mathrm{W}$ for both RF and DC, corresponding to a target power density of $3.95 \mathrm{~W} / \mathrm{cm} 2$. Thin films were deposited for 120 minutes for RF deposition and 60 minutes for DC deposition. Films were deposited using substrate temperatures of $18{ }^{\circ} \mathrm{C}$ (room temperature), $100{ }^{\circ} \mathrm{C}, 200{ }^{\circ} \mathrm{C}, 300{ }^{\circ} \mathrm{C}$ and $450{ }^{\circ} \mathrm{C}$. Film characterisation was carried out using an Ambios XP2 stylus profilometer to measure thickness, a Varian Cary 5000 spectrophotometer to measure transmission, and an Ecopia HMS 3000 Hall effect system for resistivity, carrier concentra- 
tion and mobility measurements. Transmission measurements covered the spectrum from $1500 \mathrm{~nm}$ to $200 \mathrm{~nm}$, encompassing near-infrared (IR), the visible range and the near-ultraviolet, and were taken at $5 \mathrm{~nm}$ intervals. Sheet resistance was measured using a four point probe. Resistivity measurements from the Hall device were cross-checked by multiplying the sheet resistance by the measured thickness. SEM was carried out using a Hitachi TM3030 benchtop field emission gun scanning electron microscope. The operating voltage was $5 \mathrm{kV}$. X-ray diffraction (XRD) was carried out using a Brucker D2 Phaser benchtop X-ray diffractometer equipped with a $\mathrm{Cu}-\mathrm{K}_{\alpha} \mathrm{X}$-ray gun and a Lynxeye ${ }^{\mathrm{TM}}$ detector. The beam slit was $1 \mathrm{~mm}$ wide, and the anti-scatter plate was positioned $3 \mathrm{~mm}$ above the sample. Structural characterisation was carried out on selected samples only.

\section{RESULTS AND DISCUSSION}

RF deposited films were found to be between $445 \mathrm{~nm}$ and $500 \mathrm{~nm}$ thick depending on the deposition temperature, corresponding to a deposition rate of between 0.063 and 0.069 $\mathrm{nm} / \mathrm{s}$. This is a very slow deposition rate, and would need to be significantly improved for commercial scale application. The thickness of DC deposited films was between 380 and 700, corresponding to a rate of 0.105 to $0.194 \mathrm{~nm} / \mathrm{s}$. Thus the DC deposition rate is roughly twice that for RF deposition, making it a more industrially attractive technique. The significant variation in thickness for DC deposition was unexpected as the thickness of sputtered films is typically a function of deposition time. It was found that whilst the thickness of RF deposited films was more or less constant, that of the DC deposited films decreased approximately linearly with increasing deposition temperature (Figure 11). The reason for this variation is not known.

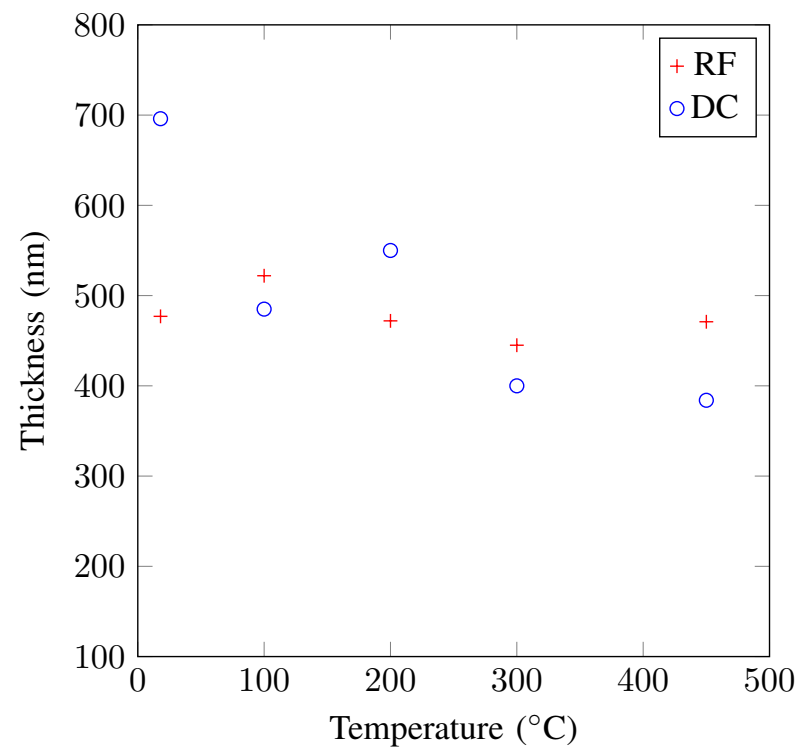

Fig. 1. Thickness against deposition temperature for both RF and DC films

Film transmission was found to be good for both deposition techniques, with all films except those deposited using DC at room temperature showing visible range transmissions of 79 $\%$ or more (Figure 2).
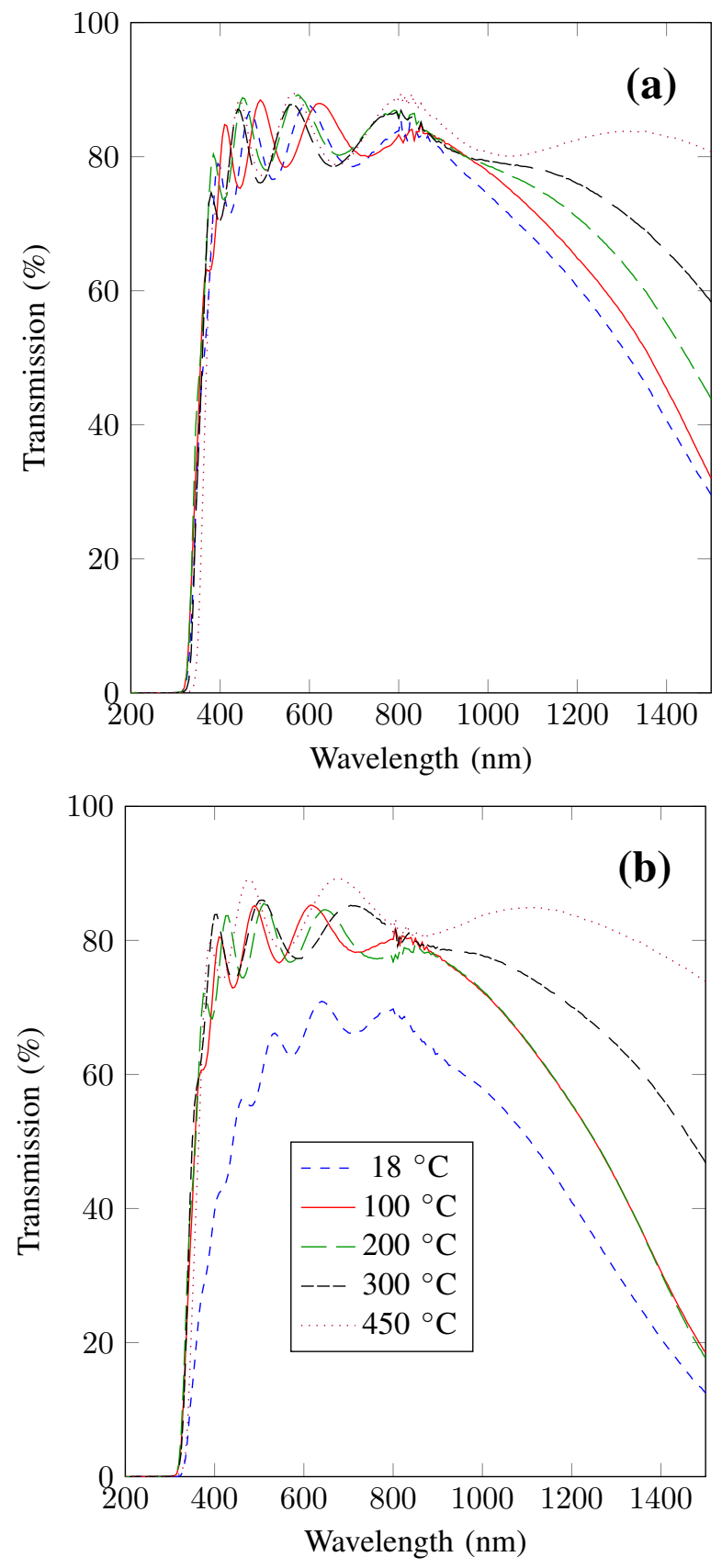

Fig. 2. Transmission curves for RF depositions (a) and DC depositions (b)

Room temperature DC films showed a rather poor average visible transmission of around $41 \%$. Average infrared (IR) transmission was found to increase with increasing temperature, which implies either a significant increase in mobility or a drop in carrier concentration [3], [8].

The film electrical properties were found to follow the same set of trends for both power supplies. Resistivity was found decrease with the initial increase in temperature. Temperature increases above $200{ }^{\circ} \mathrm{C}$ resulted in a significant increase in 
resistivity (Figure 3). The reason for the initial decrease is thought to be a result of significantly improved crystallinity with higher temperatures (eg, [9]).
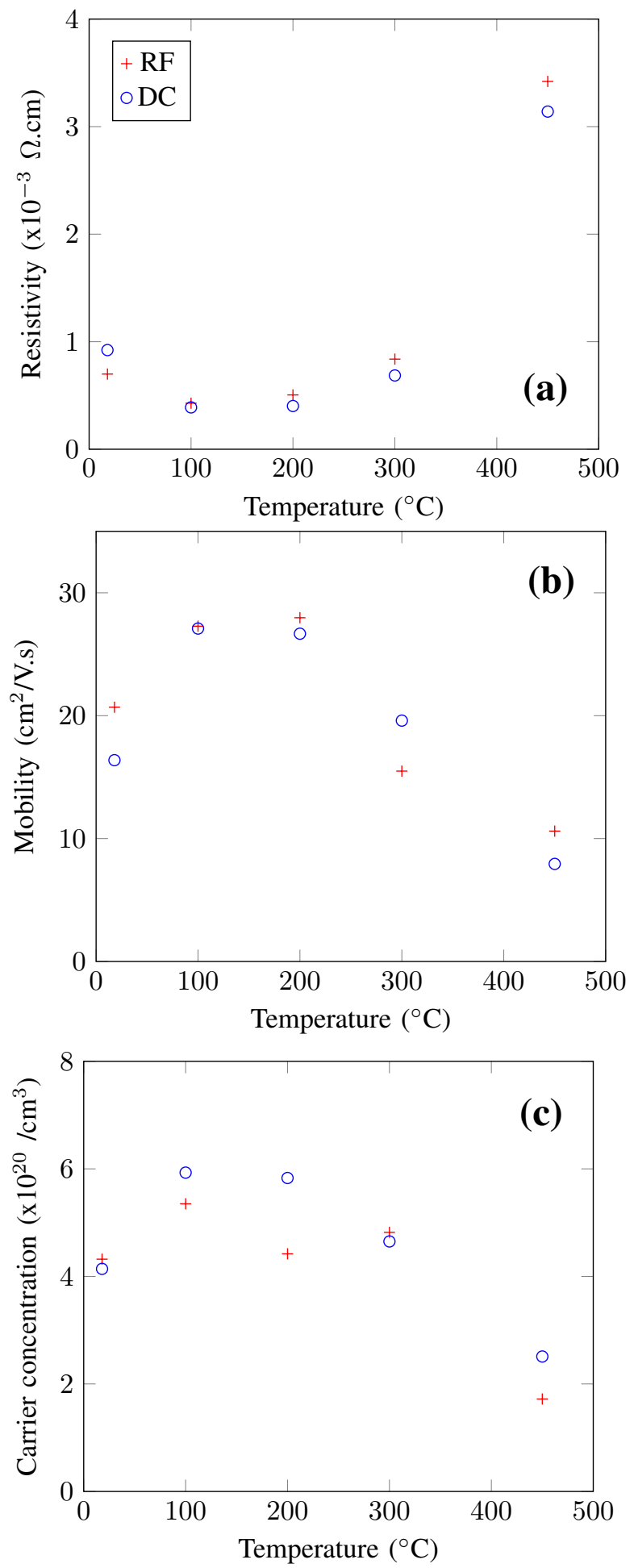

Fig. 3. Resistivity (a), mobility (b) and carrier concentration (c) against deposition temperature

Carrier concentration and mobility were found to show similar trends, with increases corresponding to the initial rise in deposition temperature followed by significant reductions as temperature was increased above $200{ }^{\circ} \mathrm{C}$. This indicates that the increase in average IR transmission is at least partly a result of reduced carrier concentration [3], [8] (Figure 4). Sheet resistance was found to follow the same trend as the resistivity, reducing slightly with the initial temperature rise before increasing significantly at higher temperatures (Figure 5).

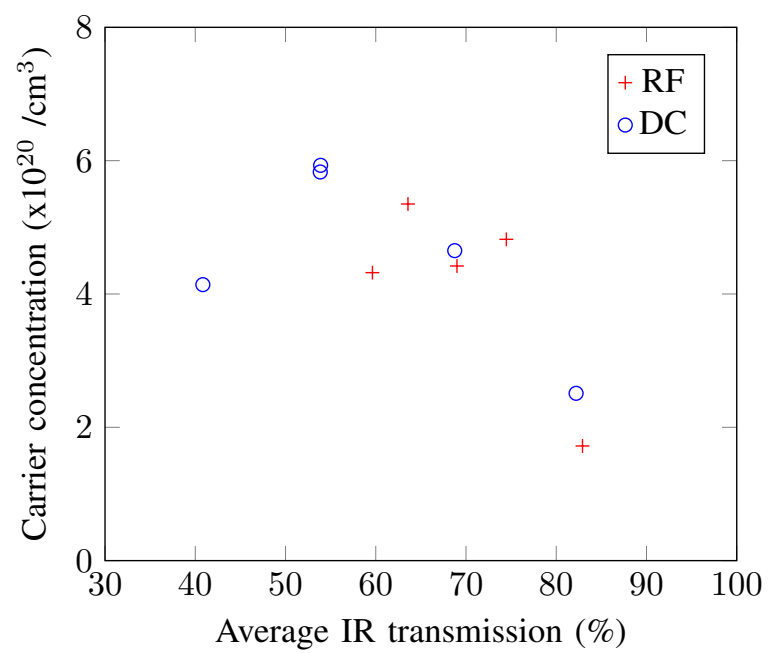

Fig. 4. Carrier concentration against average IR transmission for both RF and DC films

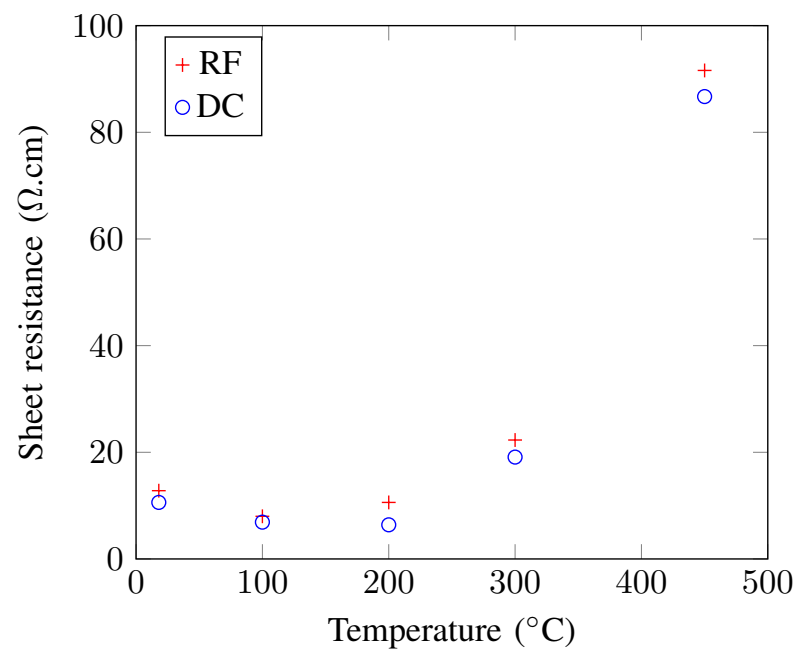

Fig. 5. Sheet resistance against deposition temperature for both RF and DC films

Dramatic increases in resistivity and IR transmission have been identified previously in sputtered AZO (eg, [10], [11]). This change is thought to be a result of reduced crystal quality with high temperature. Unfortunately due to the low maximum magnification of the SEM used it was not possible to observe the degree of crystallinity of the various films. It is clear that whatever the cause, the effect is not limited to 
RF films but occurs regardless of the type of power supply. SEM images show that the films are smooth and attain more or less complete coverage, with few if any visible pinholes. Some wider-scale surface variation and patterning is visible at lower magnifications in some areas of the films (Figure 6). Without access to a higher-powered microscope, the cause of these patterns could not be determined.
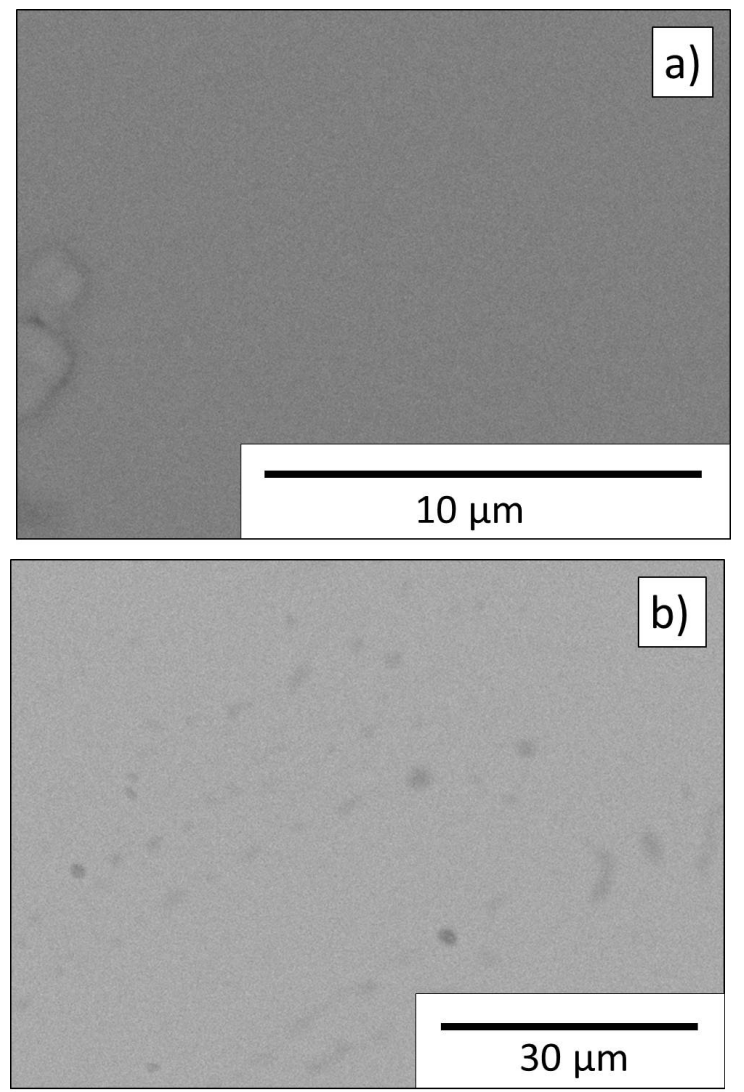

Fig. 6. SEM images of films taken at 10,000 times magnification (a) and 2,000 times magnification (b)

All thin film XRD patterns were found to be identical, with a single large peak at a $2 \Theta$ angle of $34^{\circ}$ and a second minor peak at $73^{\circ}$. These correspond to the (002) and (004) planes respectively, and is a typical pattern for sputtered zinc oxide films (Figure 7).

The significant changes in resistivity and increase in transmission with increased temperature show that deposition temperature is a key parameter in determining the material properties. That the low transmission in material deposited at room temperature is only seen in DC films means that for applications where elevated temperatures could significantly impair function, RF deposition would be preferable despite the reduced deposition rate. For applications where temperature is not a significant concern however, the significantly increased deposition rate provided by DC sputtering means that this is likely to be a more industrially appropriate deposition technique.
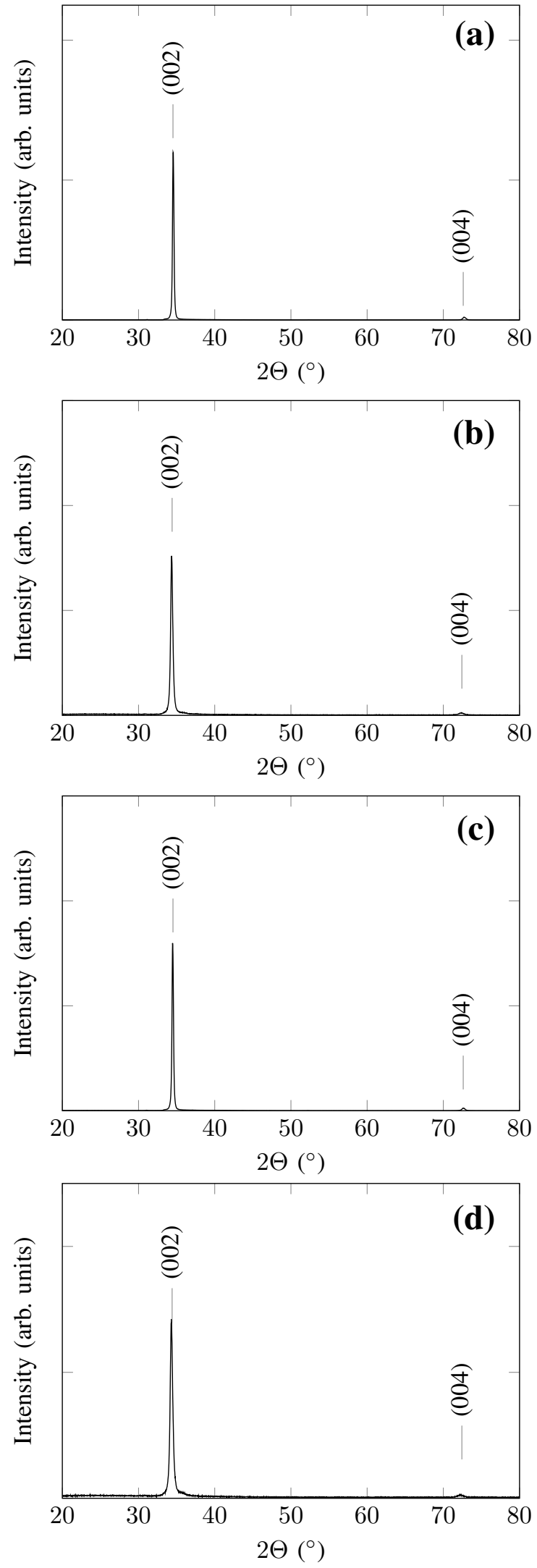

Fig. 7. XRD pattern typical of $\mathrm{ZnO}$ : RF films deposited at $100{ }^{\circ} \mathrm{C}$ (a) and $450{ }^{\circ} \mathrm{C}$ (b) and DC films deposited at $100{ }^{\circ} \mathrm{C}$ (c) and $450{ }^{\circ} \mathrm{C}$ (d) 


\section{Conclusion}

DC sputtering was found to provide roughly twice the deposition rate obtained by RF sputtering, although film thickness was found to reduce significantly with increased temperature. RF film thickness was found to be roughly constant regardless of deposition temperature. The DC process was found to be completely stable, with no indication of arcing or other significant issues.

With the exception of room temperature DC depositions, transmission for all films was found to have an average of 79 $\%$ or more in the visible range. Low temperature depositions using RF sputtering were found to show significantly higher transmission than those deposited by DC sputtering, being equivalent to higher temperature films. At more elevated temperatures, the transmission of films deposited by DC and RF were broadly similar.

Films deposited at substrate temperatures of $200{ }^{\circ} \mathrm{C}$ or less all exhibited reasonably low resistivities. Higher deposition temperatures were found to result in increased resistivity. The cause was found to be reduction in both carrier concentration and mobility. The reduced carrier concentration could also be seen in the increased IR transmission with increased substrate temperature. It is likely that the higher substrate temperature results in reduced crystal quality, which in turn is responsible for the observed deterioration in the electrical properties. XRD data showed the same patterns for all films, indicating that even the highest temperature films were crystalline. All films showed the typical zinc oxide XRD pattern. Unfortunately the SEM maximum magnification was insufficient to observe the film crystallinity.

The combination of higher deposition rates and equivalent film quality means that DC sputtering has significant promise as an industrially applicable technique for the deposition of AZO. For temperature-sensitive applications where room temperature deposition is required however, RF sputtering is more likely to provide the necessary transmission and resistivity.

\section{ACKNOWLEDGMENT}

The authors would like to acknowledge funding for the work through the EPSRC Supergen Supersolar Hub.

\section{REFERENCES}

[1] B. G. Lewis and D. C. Paine, "Applications and Processing of Transparent Conducting Oxides", MRS Bulletin vol. 25, pp. 22-27, 2000.

[2] S. Fernández and F. B. Naranjo, "Optimization of aluminum-doped zinc oxide films deposited at low temperature by radio-frequency sputtering on flexible substrates for solar cell applications", Solar Energy Materials and Solar Cells vol. 94, pp. 157-163, 2010.

[3] D. S. Ginley, H. Hosono and D. C. Paine, Handbook of Transparent Conductors, London: Springer, 2010.

[4] J-Y. Lee, S. T. Connor, Y. Cui, and P. Peumans, "Solution-processed metal nanowire mesh transparent electrodes", Nano letters vol. 8, pp. 689-692, 2008.

[5] S. Suzuki, T. Miyata, M. Ishii, and T. Minami, "Transparent conducting Vco-doped AZO thin films prepared by magnetron sputtering", Thin Solid Films vol. 434, pp. 14-19, 2003

[6] D. S. Ginley, "Nanoparticle Derived Contacts for Photovoltaic Cells", Electrochemical Society Proceedings vol. 99, pp. 103-110, 1999.

[7] C. G. Granqvist, "Transparent conductors as solar energy materials: A panoramic review", Solar Energy Materials and Solar Cells vol. 91, pp. $1529-1598,2007$
[8] S. Calnan and A. N. Tiwari, "High mobility transparent conducting oxides for thin film solar cells", Thin Solid Films vol. 518, pp. 1839-1849, 2010

[9] P. Yao, S. Hang, and M. Wu, "Growth characteristics and properties of Al-doped $\mathrm{ZnO}$ thin films by DC magnetron sputtering from AZOY(R) target”, Transactions of the Canadian Society for Mechanical Engineering, vol. 37, pp. 303-312, 2013.

[10] P. J. M. Isherwood, N. Neves, J. W. Bowers, P. Newbatt and J. M. Walls, "High quality aluminium doped zinc oxide target synthesis from nanoparticulate powder and characterisation of sputtered thin films", Thin Solid Films vol. 566, pp. 108-114, 2014.

[11] J. H. Park, J. M. Shin, S-Y. Cha, J. W. Park, S-Y. Jeong, H. K. Pak and C-R. Cho, "Deposition-Temperature Effects on AZO Thin Films Prepared by RF Magnetron Sputtering and Their Physical Properties", Jounal of the Korean Physical Society vol. 49, pp. 584-588, 2006 